\title{
Results-Healthcare Professional Interviews with Thematic Analyses
}

Eight interviews with health care professionals were conducted. This was composed of three urology specialist nurses, two oncology specialist nurses, one oncologist and two urologists. A framework analysis approach was used to analyse results (Tables 60.1, 61.1, 62.1, 63.1 and 64.1). Transcripts were reviewed several times and using a highlighter, codes and themes were identified before the final analysis was drawn. After research team familiarization with data and transcripts, the initial codes were generated manually and tabulated, using a framework analysis. All codes relating to the topic of robotic surgery for prostate cancer and psychosexual care were identified. Within these codes the over-arching themes were identified and named. Patient focus group recordings were independently reviewed by members of the research team to validate codes and themes drawn. Anonymized patient focus group results were disseminated to patients via email for patient consensus validation prior to pathway development.

This was manually done using a highlighter in a tabulated approach. Recordings of healthcare professional interviews were reviewed independently by two members of the research team for validation of codes and themes. Findings were also emailed anonymously to participants for validation. There is however, a risk, that with so many interviews, a saturation point may have been reached (Tables 60.1, 61.1, 62.1, 63.1 and 64.1). 